\title{
Article
}

Subscriber access provided by King Abdullah University of Science and Technology Library

\section{Superior Properties of Energetically Stable La2/3Sr1/3MnO3/ Tetragonal BiFeO3 Multiferroic Superlattices}

Nan Feng, Wenbo Mi, Xiaocha Wang, Yingchun Cheng, and Udo Schwingenschlögl

ACS Appl. Mater. Interfaces, Just Accepted Manuscript • DOI: 10.1021/acsami.5b02436 • Publication Date (Web): 30 Apr 2015

Downloaded from http://pubs.acs.org on May 5, 2015

\section{Just Accepted}

"Just Accepted" manuscripts have been peer-reviewed and accepted for publication. They are posted online prior to technical editing, formatting for publication and author proofing. The American Chemical Society provides "Just Accepted" as a free service to the research community to expedite the dissemination of scientific material as soon as possible after acceptance. "Just Accepted" manuscripts appear in full in PDF format accompanied by an HTML abstract. "Just Accepted" manuscripts have been fully peer reviewed, but should not be considered the official version of record. They are accessible to all readers and citable by the Digital Object Identifier (DOI囚). "Just Accepted" is an optional service offered to authors. Therefore, the "Just Accepted" Web site may not include all articles that will be published in the journal. After a manuscript is technically edited and formatted, it will be removed from the "Just Accepted" Web site and published as an ASAP article. Note that technical editing may introduce minor changes to the manuscript text and/or graphics which could affect content, and all legal disclaimers and ethical guidelines that apply to the journal pertain. ACS cannot be held responsible for errors or consequences arising from the use of information contained in these "Just Accepted" manuscripts. 


\section{Superior Properties of Energetically Stable $\mathrm{La}_{2 / 3} \mathrm{Sr}_{1 / 3} \mathrm{MnO}_{3} /$ Tetragonal}

\section{$\mathrm{BiFeO}_{3}$ Multiferroic Superlattices $^{2}$}

Nan Feng ${ }^{1}$, Wenbo $\mathrm{Mi}^{1, *}$, Xiaocha Wang ${ }^{2}$, Yingchun Cheng ${ }^{3,4}$, Udo

Schwingenschlögl ${ }^{4, *}$

${ }^{1}$ Tianjin Key Laboratory of Low Dimensional Materials Physics and Preparation Technology, Faculty of Science, Tianjin University, Tianjin 300072, China

${ }^{2}$ Tianjin Key Laboratory of Film Electronic \& Communicate Devices, School of Electronics Information Engineering, Tianjin University of Technology, Tianjin 300384, China

${ }^{3}$ Department of Mechanical and Industrial Engineering, University of Illinois at Chicago, Chicago, Illinois 60607, USA

${ }^{4}$ PSE Division, KAUST, Thuwal 23955-6900, Kingdom of Saudi Arabia 


\begin{abstract}
The superlattice of energetically stable $\mathrm{La}_{2 / 3} \mathrm{Sr}_{1 / 3} \mathrm{MnO}_{3}$ and tetragonal $\mathrm{BiFeO}_{3}$ is investigated by means of density functional theory. The superlattice as a whole exhibits a half-metallic character, as is desired for spintronic devices. The interfacial electronic states and exchange coupling are analyzed in details. We demonstrate that the interfacial $\mathrm{O}$ atoms play a key role in controlling the coupling. The higher ferroelectricity of tetragonal $\mathrm{BiFeO}_{3}$ and stronger response to the magnetic moment in $\mathrm{La}_{2 / 3} \mathrm{Sr}_{1 / 3} \mathrm{MnO}_{3} / \mathrm{BiFeO}_{3}$ superlattice show a strongly enhanced electric control of the magnetism as compared to the rhombohedral one. Therefore, it is particularly practical interest in the magnetoelectric controlled spintronic devices.
\end{abstract}

Keywords: Multiferroic; Electronic Structure; Magnetic Properties; Spintronics; Interfacial Coupling 


\title{
- INTRODUCTION
}

\begin{abstract}
Multiferroic materials display several long-range orders, such as ferromagnetism, ferroelectricity and ferroelasticity. Combination of the ferromagnetic and ferroelectric couplings allows the magnetization to be tuned by an electric field and vice versa. ${ }^{1-3}$ Such electric control of the magnetism has the potential applications in data storage as well as high-frequency magnetic devices with small currents and fields for operation. ${ }^{4}$ The perovskite $\mathrm{La}_{2 / 3} \mathrm{Sr}_{1 / 3} \mathrm{MnO}_{3}$ (LSMO) is a promising candidate in spintronic devices, owing to its $100 \%$ spin polarization of the charge carriers and high ferromagnetic Curie temperature of $T_{\mathrm{C}}=370 \mathrm{~K}{ }^{5}$ The charge-mediated magnetoelectric effect has been demonstrated in the epitaxial $\mathrm{LSMO} / \mathrm{Pb}\left(\mathrm{Zr}_{0.52} \mathrm{Ti}_{0.48}\right) \mathrm{O}_{3} .{ }^{6,7}$ The observed magnetoelectric coupling in the $\mathrm{LSMO} / \mathrm{BaTiO}{ }_{3}$ heterostructures has been attributed to the interfacial strains. ${ }^{8} \mathrm{BiFeO}_{3}(\mathrm{BFO})$ is the only known single-phase multiferroic material with a high ferroelectric $T_{\mathrm{C}}=1103 \mathrm{~K}$ and high G-type antiferromagnetic Néel temperature of $643 \mathrm{~K} .{ }^{9,10}$ Unfortunately, it suffers from weak magnetoelectric response and low electrical resistivity. ${ }^{11,12}$ An alternative pathway to realize the magnetoelectric control is the exchange bias when a ferromagnetic material is coupled with the antiferromagnetic BFO. ${ }^{13,14}$ Electric control exchange bias has been demonstrated for a heterostructure of LSMO and rhombohedral BFO, which may realize the low power devices with smaller size. ${ }^{15,16}$ Additionally, Béa et al. have reported that the


LSMO/BFO bilayers have a high and homogeneous resistivity state of the $\mathrm{BFO}$ as a ferroelectric tunnel barrier. ${ }^{17}$ Jilili et al. have found that the LSMO/rhombohedral BFO superlattice maintains the half-metallic character of bulk LSMO. ${ }^{18}$ Moreover, Guo et al . have discovered that the anomalies in the temperature-dependent ferromagnetic resonance spectra of LSMO occur at the transition temperature of Fe polarization, indicating a strong interfacial coupling between $\mathrm{Fe}$ and $\mathrm{Mn} .{ }^{19} \mathrm{Yu}$ et al. have reported a significant exchange bias for the coupling between LSMO and rhombohedral BFO using the X-ray circular dichroism method. ${ }^{20}$ Concerning the origin of the interfacial Fe magnetic ordering several physical mechanisms have been proposed, including the strong Mn-Fe hybridization, ${ }^{20} \mathrm{Fe}-\mathrm{O}-\mathrm{Fe}$ bond angle alteration and suppression of octahedral tiltings close to the interface, ${ }^{21}$ charge and orbital ordering, ${ }^{11}$ and $\mathrm{Fe} / \mathrm{Mn}$ intermixing. $^{22}$

It is found that the spontaneous polarization of tetragonal $\mathrm{BFO}$ is $150 \mu \mathrm{C} / \mathrm{cm}^{2}$, which is much higher than the value of the rhombohedral structure $\left(90 \mu \mathrm{C} / \mathrm{cm}^{2}\right){ }^{23,24}$ Zhang et al. have experimentally evidenced that ferroelectric polarization of tetragonal BFO grown on $\mathrm{LaAlO}_{3}$ substrate is significantly enhanced and have attributed it to pronounced the strain-induced Fe displacements relative to the $\mathrm{O}$ octahedron. ${ }^{25}$ Sun et al. have reported that a significantly enhanced exchange bias in bilayers of $\mathrm{Fe}_{3} \mathrm{O}_{4}$ and tetragonal $\mathrm{BFO}$ and have attributed to stronger $\mathrm{Fe}-\mathrm{O}-\mathrm{Fe}$ interfacial superexchange in the distorted structure. ${ }^{26}$ If the high spin-polarized LSMO deposited on the tetragonal 
BFO epitaxially, a strongly enhanced electric control of the magnetism may be realized, which has great potential applications in the spintronic devices. Thus, in this work we study the interfacial electronic and magnetic properties of the LSMO/tetragonal BFO superlattices by density functional theory, demonstrating a more remarkable magnetoelectric effect.

\section{- CALCULATION DETAILS AND MODELS}

We perform the density functional theory ${ }^{27}$ calculations using the projector augmented wave method ${ }^{28}$ and Perdew-Burke-Ernzerhof ${ }^{30}$ spin-polarized generalized $^{2}$ gradient approximation (GGA) as implemented in the Vienna Ab-initio Simulation Package $^{29}$. To account for the localized $d$ orbitals of the transition metals, we consider an on-site Coulomb interaction $U$. We set $U=2 \mathrm{eV}$ for $\mathrm{Mn}$ and $U=4.5 \mathrm{eV}$ for Fe, based on the previous theoretical studies for bulk LSMO and BFO. ${ }^{13,14,31}$ We have confirmed that the adopted GGA functional can give reliable describing about the half-metallicity of $\mathrm{LSMO}^{32}$ as well as anti-ferromagnetic ordering, ferroelectricity and band gap of the BFO. We employ the valence electron configurations: O $2 s^{2} 2 p^{4}$, Mn $3 d^{6} 4 s^{1}$, Fe $3 d^{7} 4 s^{1}$, $\operatorname{Sr} 4 s^{2} 4 p^{6} 5 s^{2}$, La $5 p^{6} 5 d^{1}$, and $\operatorname{Bi} 6 s^{2} 6 p^{3}$. The calculations are performed with a plane wave cutoff energy of $500 \mathrm{eV}$, where $\Gamma$-centered $9 \times 9 \times 9$ and $9 \times 9 \times 1 \mathrm{k}$-meshes are used for the bulk compounds and the superlattice, respectively. The convergence criterion 
for the energy is set to $10^{-5} \mathrm{eV}$ and that for the atomic forces to $0.01 \mathrm{eV} / \AA$.

Tetragonal BFO with space group $P 4 m m$ has a lattice constant of $a=3.94 \AA$ and $c / a=1.02,{ }^{33,34}$ where the $\mathrm{O}_{\mathrm{A}}$ and $\mathrm{O}_{\mathrm{B}}$, respectively, belong to the $\mathrm{BiO}$ and $\mathrm{FeO}$ layers, see Figure 1a. It is not clear which antiferromagnetic order of G-type and C-type in the tetragonal phase film would show. ${ }^{35}$ In this work, we consider G-type antiferromagnetism, in which the magnetic moments of $\mathrm{Fe}$ atoms align ferromagnetically in (111) planes and antiferromagnetically between adjacent (111) planes. ${ }^{36}$ Bulk LSMO has a tetragonal structure (space group $P 4 m m$ ) with the lattice constant $a=3.88 \AA$ and $c / a=3.00 .{ }^{37}$ The unit cell contains four layers: SrO, LaO,

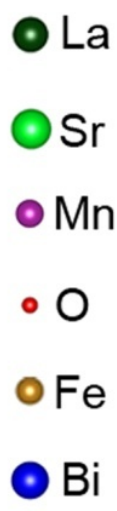

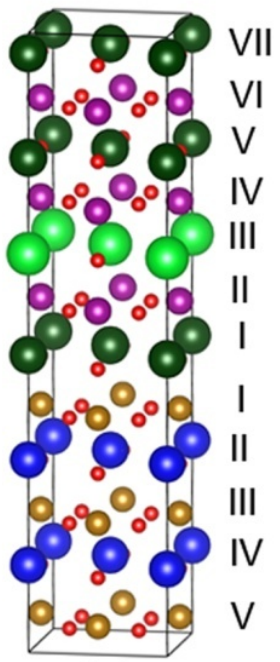

(c)

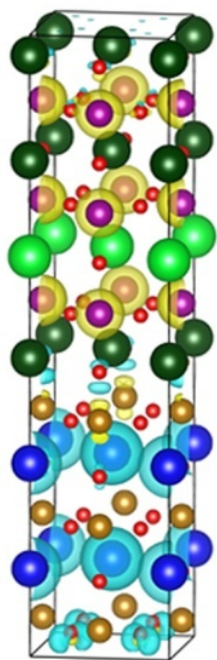

(d)

Figure 1. (Color online) Structures of (a) bulk BFO, (b) bulk LSMO, and (c) the LSMO/BFO superlattice. (d) Charge density difference (isosurface value $0.01 \mathrm{e} / \AA^{3}$ ) for the LSMO/BFO superlattice relative to the two components. Yellow regions represent the charge accumulation and blue regions charge depletion. 
$\mathrm{Mn}_{\mathrm{A}} \mathrm{O}_{2}$ and $\mathrm{Mn}_{\mathrm{B}} \mathrm{O}_{2}$, see Figure $1 \mathrm{~b}$. The $\mathrm{Mn}_{\mathrm{A}}$ atom sees a $\mathrm{SrO}$ layer below and a $\mathrm{LaO}$ layer on top, whereas the $\mathrm{Mn}_{\mathrm{B}}$ atom sees $\mathrm{LaO}$ layers on both sides. We model the superlattice using a slab geometry with seven LSMO and five BFO layers. And it is based on an $\sqrt{2} \times \sqrt{2}$ in-plane supercell for accommodating the BFO's G-type antiferromagnetic ordering. The surface unit cell of $\mathrm{BFO}(001)$ has $\sqrt{2} \times \sqrt{2} R 45^{\circ}$ periodicity with lattice constant $5.57 \AA$, while the surface unit cell of $\operatorname{LSMO}(001)$ has the same periodicity with a lattice constant of $5.49 \AA$, i.e., the lattice mismatch is less than $1.5 \%$. This allows us to use the average lattice constant for its ab-plane.

Eight stacking patterns with different terminations of LSMO on BFO can be realized: $\mathrm{LSMO}(001)$ with $\mathrm{SrO}, \mathrm{LaO}, \mathrm{Mn}_{\mathrm{A}} \mathrm{O}_{2}$, and $\mathrm{Mn}_{\mathrm{B}} \mathrm{O}_{2}$ terminations and $\mathrm{BFO}(001)$ with $\mathrm{BiO}_{\mathrm{A}}$ and $\mathrm{Fe}\left(\mathrm{O}_{\mathrm{B}}\right)_{2}$ terminations. As of the periodic boundary conditions are applied in all the directions, two interfaces are present in each supercell, which are inequivalent in an asymmetric structure.

\section{- RESULTS AND DISCUSSION}

The most stable structure is determined by calculating the work of separation, i.e., the cohesive energy between LSMO and BFO, $W_{\text {sep }}=\left(E_{L S M O / B F O}-E_{L S M O}-E_{B F O}\right) / 2$, where $E_{L S M O / B F O}$ is the total energy of the superlattice and $E_{L S M O}$ and $E_{B F O}$ are the energies of the same supercell containing only relaxed LSMO and BFO, respectively. 
Table 1. Average vertical distances $d_{z}$ and $d_{z^{\prime}}$ (in $\AA$ ) of the $\mathrm{O}$ plane relative to its cationic plane in the BFO and LSMO regions of the tetragonal LSMO/BFO superlattice, see Figure 1c. Positive/negative numbers denote shifts towards/off the Fe atoms in layer I. Comparison of the calculated magnetic moments (in $\mu_{\mathrm{B}}$ ) of $\mathrm{Mn}$ and $\mathrm{O}$ atoms near the interfaces in LSMO/BFO superlattice with tetragonal or rhombohedral BFO.

\begin{tabular}{cccccccccc}
\hline \hline System & & Bulk & I & II & III & IV & V & VI & VII \\
\hline \multirow{5}{*}{ Tetragonal } & $d_{\mathrm{z}}$ & $-0.61 /-0.79$ & -0.02 & -0.45 & -0.28 & -0.43 & -0.35 & - & - \\
& $d_{z^{\prime}}$ & 0 & +0.49 & +0.28 & +0.19 & -0.07 & +0.07 & +0.14 & 0 \\
& $\mathrm{Mn}$ & $3.42 / 3.68$ & - & 3.84 & - & - & - & 3.23 & - \\
& $\mathrm{O}$ & 0.01 & -0.11 & -0.01 & - & - & - & -0.02 & 0 \\
\multirow{5}{*}{ Rhombohedral } & $\mathrm{Mn}$ & $3.42 / 3.68$ & - & 3.47 & - & - & - & 3.52 & - \\
& $\mathrm{O}$ & 0.01 & -0.07 & -0.02 & - & - & - & -0.01 & 0.05 \\
\hline \hline
\end{tabular}

The factor $1 / 2$ accounts for the two interfaces per supercell. The energetically favorable configuration after the structural optimization, see Figure 1c, is LaO-terminated with interfacial $\mathrm{O}$ atoms located on top of interfacial Fe atoms and has $W_{\text {sep }}=5.13 \mathrm{eV}$. This is consistent with the previous findings for the supercell consisting of LSMO and rhombohedral $\mathrm{BFO}^{18,22}$ and is also in a similar $\mathrm{LSMO} / \mathrm{YMnO}_{3}$ system, ${ }^{38}$ in which the LaO-terminated interface has always the lowest energy. The largest work of separation suggests that this configuration is the most stable structure that occurs during the growth. The average interplanar spacing of the $\mathrm{LaO}$ and $\mathrm{FeO}_{2}$ layers (both labeled $\mathrm{I}$ in Figure 1c) is $1.93 \AA$ and that of the $\mathrm{LaO}$ and $\mathrm{FeO}_{2}$ layers (labeled VII and V in Figure 
1c) is $1.56 \AA$. More specifically, the $\mathrm{O}$ atoms are shifted out of the cationic plane, see Table 1. For BFO region, the relative Fe-O and Bi-O displacements are much smaller than the ferroelectric displacement in bulk $\mathrm{BFO}$, where the $\mathrm{Fe}-\mathrm{O}$ and $\mathrm{Bi}-\mathrm{O}$ displacements in bulk BFO along [001] direction are 0.61 and $0.79 \AA$, respectively. Therefore, the BFO is subjected to a reduced ferroelectric polarization. Furthermore, the $\mathrm{O}$ atoms in $\mathrm{BFO}$ region are displaced away from layer $\mathrm{I}$, indicating a net polarization in BFO pointing to layer V. As for LSMO region, in layer I they shift heavily by $0.49 \AA$ towards Fe atoms, whereas layer VII stays almost planar. As a result, the Fe-O bond lengths through the two interfaces amount to 1.93 and $2.31 \AA$, respectively. The former is slightly shorter than bulk FeO $(2.16 \AA),{ }^{39}$ indicative of a relatively strong interaction between the adjacent $\mathrm{Fe}$ and $\mathrm{O}$ atoms. The huge shifts of the $\mathrm{O}$ atoms can be expected to determine the interfacial properties, since these atoms mediate the interaction between LSMO and BFO.

Figures $2 \mathrm{a}$ and $\mathrm{b}$ show the total and projected densities of states (DOS) of fully relaxed bulk BFO and LSMO, respectively. For BFO the calculated band gap of 1.90 $\mathrm{eV}$ is in excellent agreement with previous calculations ${ }^{13}$ but does not reach the experimental value of $2.50 \mathrm{eV},{ }^{40}$ due to the limitations of the local exchange and correlation functional on reproducing the derivative discontinuity. ${ }^{41} \mathrm{Bi} s$ states has negligible contribution to the bonding. The Fe spins are directed antiparallel but the DOS is the same, so we show only one atom. The calculated Fe magnetic moment in 

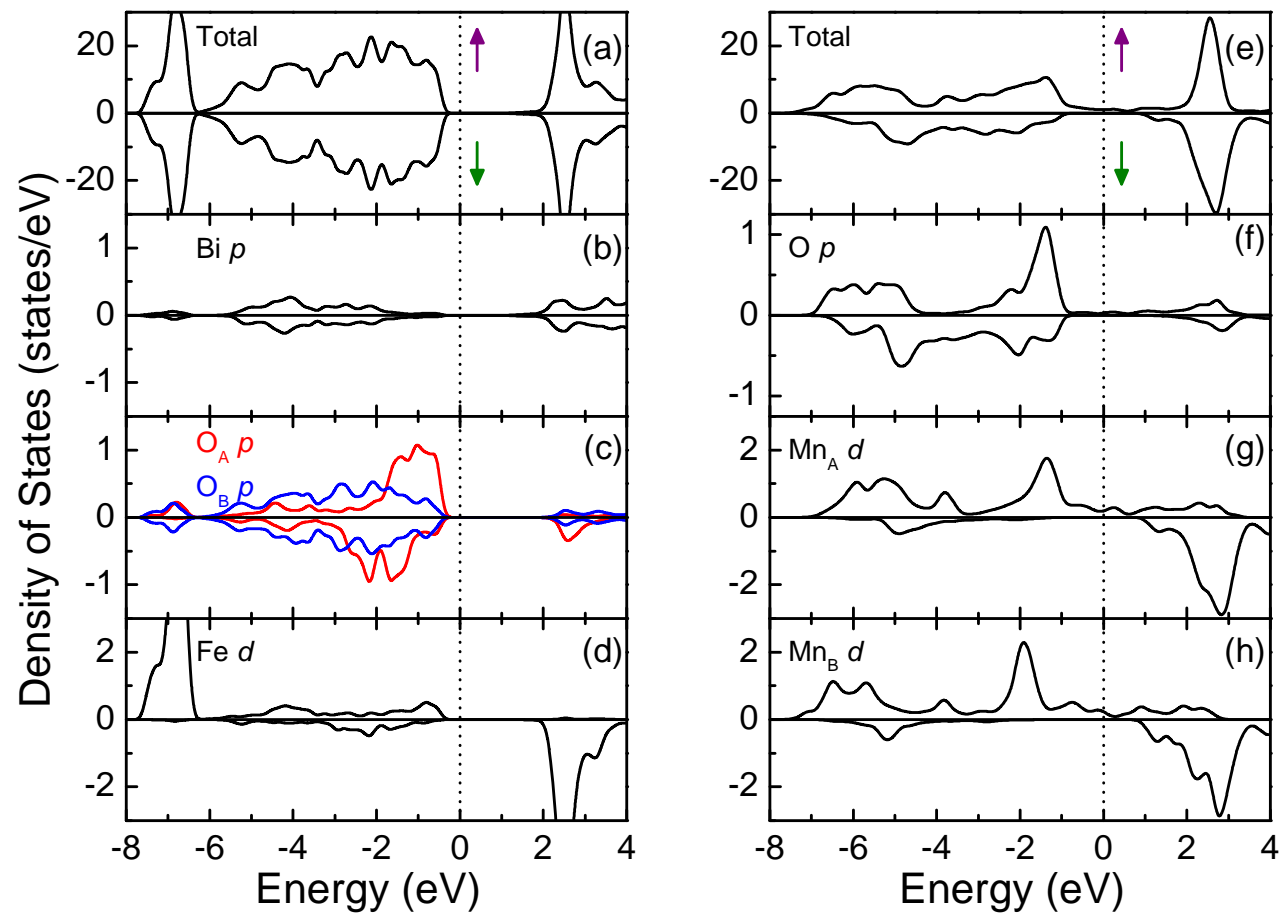

Figure 2. (Color online) Total and PDOS of bulk BFO (a-d) and bulk LSMO (e-h). The Fermi energy is indicated by vertical dashed lines and is set to zero.

bulk BFO is $\pm 4.18 \mu_{\mathrm{B}} /$ atom, agreeing well with a previously reported value. ${ }^{33}$ The LSMO DOS exhibits a half-metallic character with a spin-down band gap of $1.87 \mathrm{eV}$. The magnetic moments of 3.42 and $3.68 \mu_{\mathrm{B}}$ are obtained for $\mathrm{Mn}_{\mathrm{A}}$ and $\mathrm{Mn}_{\mathrm{B}}$, respectively. These values are close to experimental value of $3.70 \mu_{\mathrm{B}}{ }^{42}$ These results confirm that the rationality of the adopted parameters used in the present calculations.

The band structure, partial DOS and charge density difference obtained for the energetically favorable configuration of the superlattice are addressed in Figures 3, 4 and 1d, respectively. In Figure 3 the red and blue dots represent contributions of 

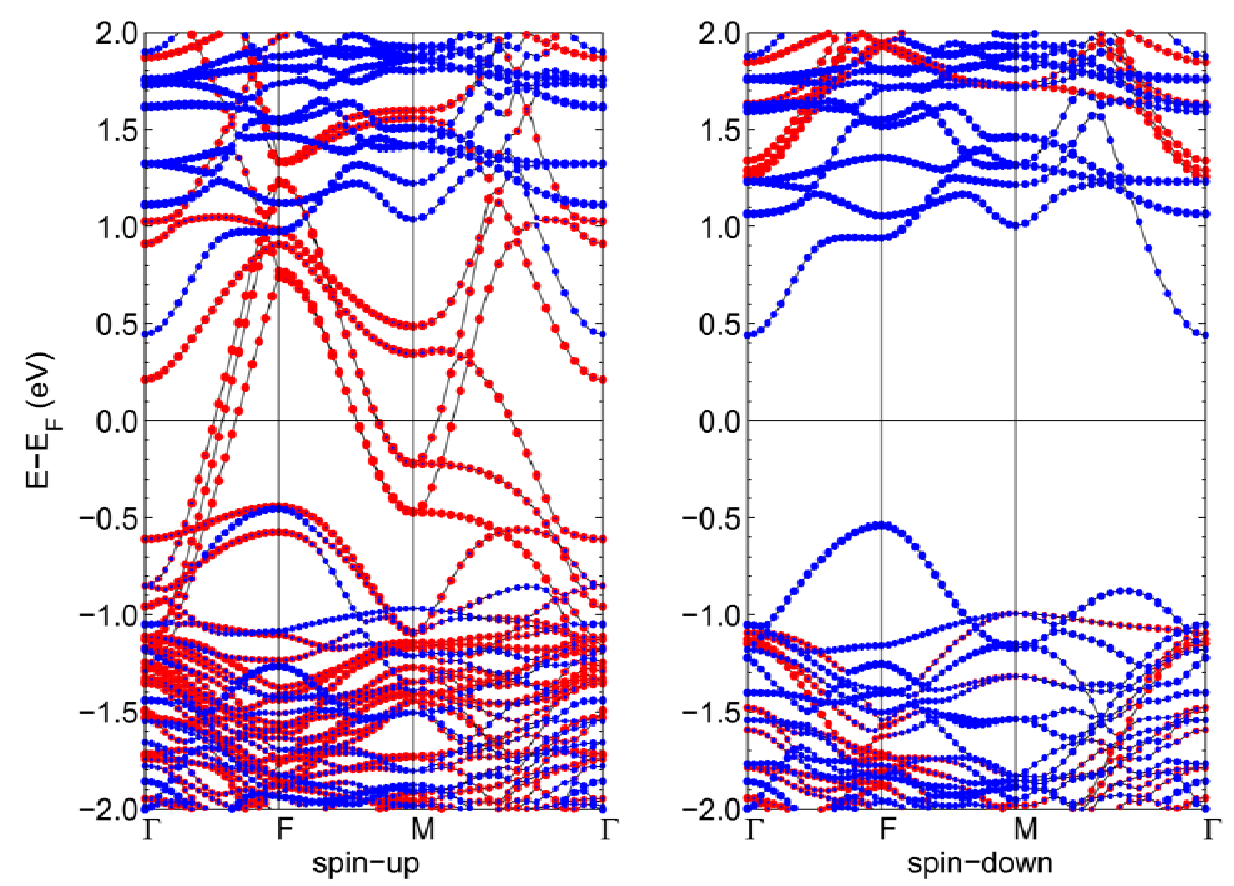

Figure 3. (Color online) Spin-up and spin-down band structures of the LSMO/BFO superlattice. The size of the blue dots is proportional to the BFO contribution, whereas that of the red dots is proportional to the LSMO contribution.

LSMO and BFO to the eigenstates, respectively, where the size quantifies the contribution. For BFO, the spin-up bands cross the Fermi level, exhibiting a metallic character, while the spin-down channel is semiconducting with a band gap of $0.98 \mathrm{eV}$, constituting a half-metallic character. LSMO is also half-metallic with a spin-down band gap of $2.3 \mathrm{eV}$. Thus, the entire superlattice is half-metallic, as is desired for the spintronic devices.

As compared to bulk LSMO, the magnetic moments in the superlattice decrease slightly. Since the bulk-like inner atomic layers nicely reproduce the bulk properties, 
we focus on the atoms close to the interface. In Figure 4, the lable I-Fe, for example, refers to the Fe atom in layer I, as defined in Figure 1c. Again, we address only one of the antiferromagnetically ordered Fe atoms. For BFO, Fermi level differs from the bulk: In layer I it shifts to higher energy by $1.16 \mathrm{eV}$, while in layer $\mathrm{V}$ it is not shifted. The Fermi level upshift makes the orbital energies of $\mathrm{Fe} d$ in layer I close to $\mathrm{O} p$ states in layer I of LSMO, which enhances the energetic overlap between them. Hence, the Fe-O hybridization through the interface appears. Although there is no direct bonding between $\mathrm{Mn}$ in layer II and $\mathrm{Fe}$ in layer I, the indirect coupling mediated by $\mathrm{O}$ in layer I
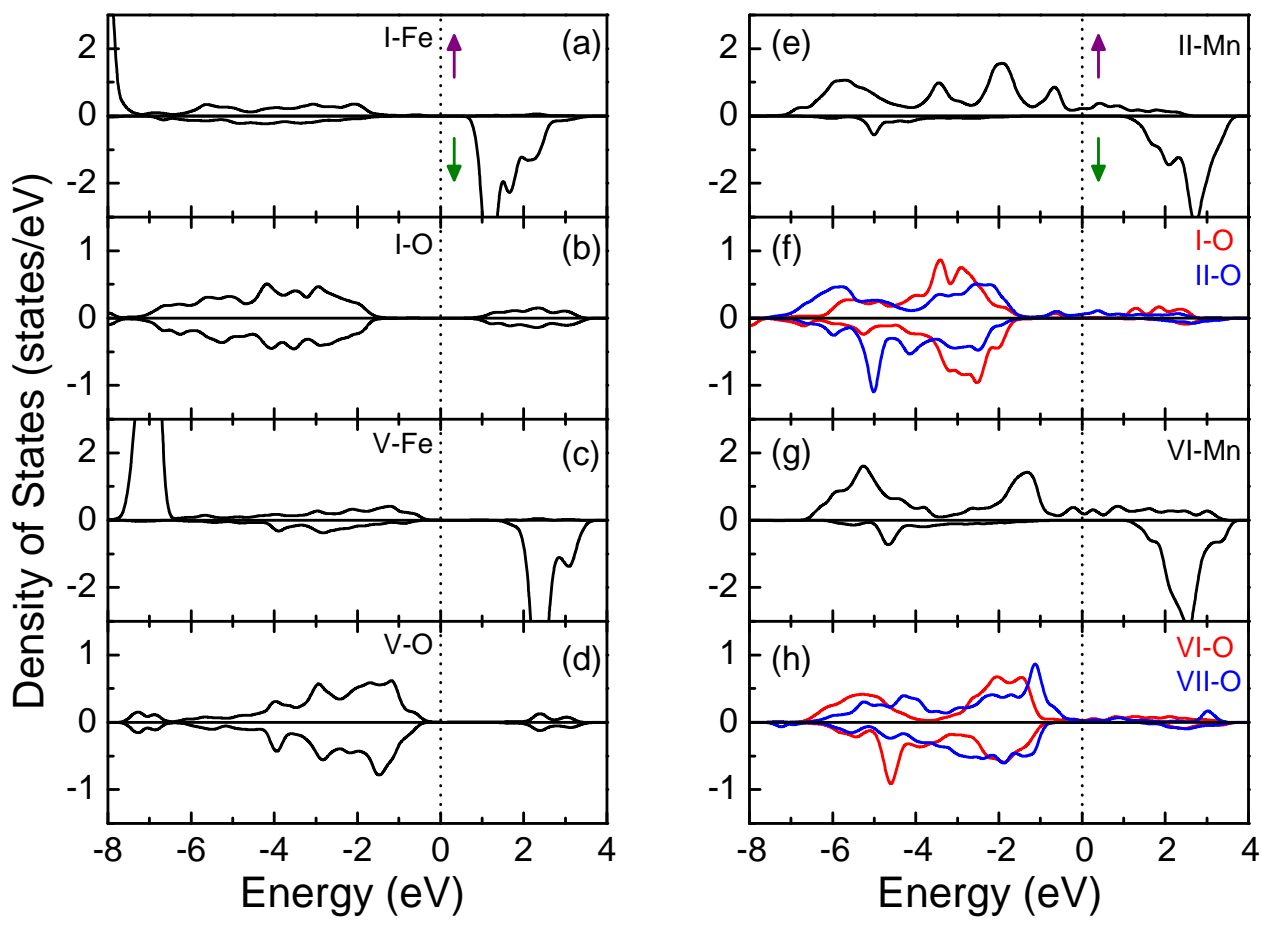

Figure 4. (Color online) PDOS for the interfacial BFO (a-d) and LSMO layers (e-h) in the fully relaxed LSMO/BFO superlattice. The Fermi energy is indicated by vertical dashed lines and set at zero. 
has a sizable effect on the $\mathrm{Mn} d$ states. The hybridization of $\mathrm{Mn}$ in layer II with $\mathrm{O}$ in layers I and II is slightly weaker than in the bulk, which enhances the magnetic moment to $3.84 \mu_{\mathrm{B}}$ and induces a huge $\mathrm{O}$ magnetic moment of $0.11 \mu_{\mathrm{B}}$ with opposite sign. We find strong hybridization between $\mathrm{Mn}$ in layer VI and O in layer VII in the whole energy range and between $\mathrm{Mn}$ in layer VI and $\mathrm{O}$ in layer $\mathrm{V}$ through the interface in valence band, as shown in Figure $4 \mathrm{~d}, \mathrm{~g}$ and $\mathrm{h}$. This reduces the Mn magnetic moment to $3.23 \mu_{\mathrm{B}}$. The hybridization between $\mathrm{O}$ and $\mathrm{Fe}$ in layers $\mathrm{I}$ and $\mathrm{V}$ does not change with respect to bulk so that the Fe magnetic moments retain their bulk values. The half-metallicity in BFO results from the contributions of the spin-up Fe $d$ states in layer $\mathrm{I}$ and $\mathrm{O} p$ states in layer $\mathrm{V}$ due to the strong hybridization with $\mathrm{O}$ in layer $\mathrm{I}$ and $\mathrm{Mn}$ in layer VI. We note that as a result of the exchange coupling, the LSMO magnetization varies significantly near the two interfaces: Mn in layer II (VI) gains a magnetic moment of $0.42(-0.45) \mu_{\mathrm{B}}$, and the change of $\mathrm{O}$ magnetic moment is -0.12 $\mu_{\mathrm{B}}$ for layer I and $-0.03 \mu_{\mathrm{B}}$ for layer VI (see Table 1). This suggests that, when an electric field is applied to this configuration so as to reverse the ferroelectric polarization of $\mathrm{BFO}$, the variation of the interfacial exchange coupling strength will modify the interfacial magnetization.

In order to judge the feasibility of the use of tetragonal BFO in the LSMO/BFO superlattice that can improve the magnetoelectric coupling, we have also analyzed the rhombohedral BFO for comparison. The rhombohedrally-distorted perovskite BFO 
(space group: $R 3 c$ ) has the lattice constants of $a=3.96 \AA$ and $\alpha=89.5^{\circ} .{ }^{43}$ For the rhombohedral BFO based superlattice, we use the same computational setup as that in the tetragonal LSMO/BFO superlattice. We find an asymmetry between Mn magnetic moments near two interfaces: 3.47 and $3.52 \mu_{\mathrm{B}}$ for layers II and VI, respectively, so that the Mn magnetic moment change is $0.05 \mu_{\mathrm{B}}$ for layer II and $-0.16 \mu_{\mathrm{B}}$ for layer VI with respect to bulk value. Jilili et al. have found that the difference in the $\mathrm{Mn}$ magnetic moments near two interfaces caused by the polarization is 0.33 and $0.38 \mu_{\mathrm{B}}$, respectively. ${ }^{18}$ Neumann et al. have found that the change is about $0.15 \mu_{\mathrm{B} .}{ }^{22}$ Importantly, we note that the use of tetragonal $\mathrm{BFO}$ has a stronger response to the variation of magnetic moment than the rhombohedral one, indicating a larger magnetoelectric effect. Our calculated work of separation for the interface between LSMO and rhombohedral BFO is $-6.12 \mathrm{eV}$, which has the same sign as the interface to the tetragonal BFO, suggesting both the interfaces are thermodynamically stable. The different phases of the BFO layer in the stack determine its ferroelectric polarization and subsequently the heterostructure properties. A high-quality tetragonal BFO film has been grown on $\mathrm{LaAlO}_{3}$ substrate by pulsed laser deposition ${ }^{25}$ or magnetron sputtering $^{26}$, so one can deposit epitaxial LSMO layers on tetragonal BFO due to the good lattice match. Stronger magnetoelectric effect in the tetragonal BFO/LSMO heterostructures will stimulate further interest in developing the magnetoelectric controlled spintronic devices. We hope that our theoretical prediction on the 
magnetoelectric effect in the tetragonal BFO/LSMO heterostructures can provide the useful information for experiments.

The detailed nature of the interaction at the LSMO/BFO interfaces is illustrated by the difference of the charge densities of the isolated LSMO and BFO slabs and that of the superlattice, see Figure 1d. We find a significant charge accumulation around Fe in layer I and around $\mathrm{Mn}$ in layer II, while $\mathrm{O}$ in layer I looses charge. The charge of O in layer $\mathrm{V}$ is redistributed to $\mathrm{O}$ in layer VII and to $\mathrm{MnO}_{2}$ in layer VI. As discussed previously, the spontaneous polarization of $\mathrm{BFO}$ region points toward layer $\mathrm{V}$. Depositing LSMO atop BFO, the polar discontinuity at the interface results in the divergence of the electrostatic potential when the number of BFO grows because of the periodic boundary conditions. Electrons in BFO are transferred across the interface into LSMO region to form a build-in electric field pointing from BFO to LSMO for avoiding the divergence. Therefore, charge accumulates in deeper Mn layers and charge depletes in deeper Bi layers. The appearance of magnetoelectric effect in the heterostructure composed of LSMO and tetragonal BFO is consistent with the experimental findings in the similar $\mathrm{LSMO} / \mathrm{Pb}\left(\mathrm{Zr}_{0.52} \mathrm{Ti}_{0.48}\right) \mathrm{O}_{3}$ and $\mathrm{LSMO} / \mathrm{BaTiO}_{3}$ heterostructures. ${ }^{6-8}$ The use of $\mathrm{BFO}$ in its tetragonal phase in LSMO/BFO heterostructure has a more remarkable magnetoelectric effect than the rhombohedral one. Meanwhile, a significantly enhanced exchange bias in bilayers of $\mathrm{Fe}_{3} \mathrm{O}_{4}$ and tetragonal BFO than that of the rhombohedral one also has been observed 
experimentally. ${ }^{26}$

\section{- CONCLUSION}

In conclusion, the density functional theory has been used to study the structural stability and electronic properties of the tetragonal LSMO/BFO(001) superlattice. The energetically favorable interface is found to consist of LaO-terminated LSMO and $\mathrm{FeO}_{2}$-terminated BFO. The superlattice presents a half-metallicity and the interfacial exchange coupling (Fe-O-Mn) increases the Mn magnetic moment and induces a huge $\mathrm{O}$ magnetic moment of $0.11 \mu_{\mathrm{B}}$. The surprising half-metallicity in the BFO domain originates from specific modifications of the orbital hybridizations due to the interaction with LSMO. It turns out that the interfacial $\mathrm{O}$ atoms and their spatial shifts play a key role in mediating the interaction and thus in the exchange mechanism. The tetragonal $\mathrm{LSMO} / \mathrm{BFO}(001)$ superlattice is characterized by a strongly enhanced electric control of the magnetism and therefore of particular practical interest in magnetoelectric controlled spintronics.

\section{- AUTHOR INFORMATION}

\section{Corresponding Author}


*E-mail: miwenbo@tju.edu.cn and Udo.Schwingenschlogl@kaust.edu.sa

Author Contributions

N.F. and W.M. designed the outline of the manuscript and wrote the main manuscript text. X.W., Y.C., and U.S. contributed detailed discussions and revisions. All authors reviewed the manuscript.

Notes

The authors declare no competing financial interest

\title{
- ACKNOWLEDGEMENTS
}

\author{
W. M. was supported by the NSFC of China (51171126), Key Project of TSTC of \\ Tianjin (12JCZDJC27100 and 14JCZDJC37800), Program for NCET in University \\ (NCET-13-0409) and Scientific Research Foundation for the Returned Overseas \\ Chinese Scholars, State Education Ministry of China. It is also supported by High \\ Performance Computing Center of Tianjin University, China. Research reported in this \\ publication was supported by the King Abdullah University of Science and Technology \\ (KAUST).
}




\section{- REFERENCES}

(1) Eerenstein, W.; Mathur, N. D.; Scott, J F. Multiferroic and Magnetoelectric Materials. Nature 2006, 442, 759-765.

(2) Fiebig, M. Revival of the Magnetoelectric Effect. J. Phys. D 2005, 38, R123-R152.

(3) Spaldin, N A.; Fiebig, M. The Renaissance of Magnetoelectric Multiferroics. Science 2005, 309, 391-392.

(4) Huijben, M.; Yu, P.; Martin, L. W.; Molegraaf, H. J. A.; Chu, Y. H.; Holcomb, M. B.; Balke, N.; Rijnders, G.; Ramesh, R. Ultrathin Limit of Exchange Bias Coupling at Oxide Multiferroic/Ferromagnetic. Adv. Mater. 2013, 25, 4739-4745.

(5) Bowen, M.; Barthélémy, A.; Bibes, M.; Jacquet, E.; Contour, J. P.; Fert, A.; Ciccacci, F.; Duò, L.; Bertacco, R. Spin-Polarized Tunneling Spectroscopy in Tunnel Junctions with Half-Metallic Electrodes. Phys. Rev. Lett. 2005, 95, 137203.

(6) Mukherjee, D.; Hordagoda, M.; Lampen, P.; Phan, M. H.; Srikanth, H. Simultaneous enhancements of polarization and magnetization in epitaxial $\mathrm{Pb}\left(\mathrm{Zr}_{0.52} \mathrm{Ti}_{0.48}\right) \mathrm{O}_{3} / \mathrm{La}_{0.7} \mathrm{Sr}_{0.3} \mathrm{MnO}_{3}$ multiferroic heterostructures enabled by ultrathin $\mathrm{CoFe}_{2} \mathrm{O}_{4}$ sandwich layers, Phys. Rev. Lett. 2015, 91, 054419.

(7) Dussan, S.; Kumar, A.; Scott, J. F.; Katiyar, R. S. Magnetic effects on dielectric and polarization behavior of multiferroic heterostructures, Appl. Phys. Lett. 2010, 96, 072904.

(8) Eerenstein, W.; Wiora, M.; Prieto, J. L.; Scott, J. F.; Mathur, N. D. Giant sharp and 
persistent converse magnetoelectric effects in multiferroic epitaxial heterostructures, Nature Mater. 2007, 6, 348-351.

(9) Choi, W. S.; Jeong, D. W.; Seo, S. S. A.; Lee, Y. S.; Kim, T. H.; Jang, S. Y.; Lee, H. N.; Myung-Whun, K. Charge States and Magnetic Ordering in $\mathrm{LaMnO}_{3} / \mathrm{SrTiO}_{3}$ Superlattices. Phys. Rev. B 2011, 83, 195113.

(10) Garcia-Barriocanal, J.; Cezar, J. C.; Bruno, F. Y.; Thakur, P.; Brookes, N. B.; Utfeld, C.; Rivera-Calzada, A.; Giblin, S. R.; Taylor, J. W.; Duffy, J. A.; Dugdale, S. B.; Nakamura, T.; Kodama, K.; Leon, C.; Okamoto, S.; Santamaria, J. Spin and Orbital Ti Magnetism at $\mathrm{LaMnO}_{3} / \mathrm{SrTiO}_{3}$ Interfaces. Nat. Commun. 2010, 1, 82.

(11) Calderón, M. J.; Liang, S.; Yu, R.; Salafranca, J.; Dong, S.; Yunoki, S.; Brey, L.; Moreo, A.; Dagotto, E. Magnetoelectric Coupling at the Interface of $\mathrm{BiFeO}_{3} / \mathrm{La}_{0.7} \mathrm{Sr}_{0.3} \mathrm{MnO}_{3}$ multilayers. Phys. Rev. B 2011, 84, 024422.

(12) Higuchi, T.; Liu, Y. S.; Yao, P.; Glans, P. A.; Guo, J.; Chang, C. L.; Wu, Z. Y.; Hattori, T. Electronic Structure of Multiferroic $\mathrm{BiFeO}_{3}$ by Resonant Soft X-Ray Emission Spectroscopy. Phys. Rev. B 2008, 78, 085106.

(13) Yang, H.; Jin, C.; Mi, W. B.; Bai, H. L.; Chen, G. F. Electronic and Magnetic Structure of $\mathrm{Fe}_{3} \mathrm{O}_{4} / \mathrm{BiFeO}_{3}$ Multiferroic Superlattices: First Principles Calculations. J. Appl. Phys. 2012, 112, 063925.

(14) Feng, N.; Mi, W. B.; Wang, X. C.; Bai, H .L. The Magnetism of $\mathrm{Fe}_{4}$ N/oxides $\left(\mathrm{MgO}, \mathrm{BaTiO}_{3}, \mathrm{BiFeO}_{3}\right)$ Interfaces from First-principles Calculations. RSC Adv. 2014, 4, 48848-48859.

(15) Wu, S. M.; Cybart, S. A.; Yu, P.; Rossell, M. D.; Zhang, J. X.; Ramesh, R.; Dynes, 
R. C. Reversible Electric Control of Exchange Bias in a Multiferroic Field-Effect Device. Nature Mater. 2010, 9, 756-761.

(16) Wu, S. M.; Cybart, S. A.; Yi, D.; Parker, J. M.; Ramesh, R.; Dynes, R. C. Full Electric Control of Exchange Bias. Phys. Rev. Lett. 2013, 110, 067202.

(17) Béa, H.; Bibes, M.; Sirena, M.; Herranz, G.; Bouzehouane, K.; Jacquet, E.; Fusil, S.; Paruch, P.; Dawber, M.; Contour, J. P.; Barthélémy, A. Combining Half-Metals and Multiferroics into Epitaxial Heterostructures for Spintronics. Appl. Phys. Lett. 2006, $88,062502$.

(18) Jilili, J.; Eckern, U.; Schwingenschlögl, U. Half-Metallicity in a $\mathrm{BiFeO}_{3} / \mathrm{La}_{2 / 3} \mathrm{Sr}_{1 / 3} \mathrm{MnO}_{3}$ Superlattice: A First-Principles Study. EPL 2013, 102, 67009.

(19) Guo, H. Y.; Lin, J. G.; Yang, J. C.; Chu, Y. H. Dynamic Magnetic Interaction in $\mathrm{La}_{2 / 3} \mathrm{Sr}_{1 / 3} \mathrm{MnO}_{3} / \mathrm{BiFeO}_{3}$ Heterostructure. Appl. Phys. Lett. 2014, 105, 112406.

(20) Yu, P.; Lee, J. S.; Okamoto, S.; Rossell, M. D.; Huijben, M.; Yang, C. H.; He, Q.; Zhang, J. X.; Yang, S. Y.; Lee, M. J.; Ramasse, Q. M.; Erni, R.; Chu, Y. H.; Arena, D. A.; Kao, C. C.; Martin, L. W.; Ramesh, R. Interface Ferromagnetism and Orbital Reconstruction in $\mathrm{BiFeO}_{3}-\mathrm{La}_{0.7} \mathrm{Sr}_{0.3} \mathrm{MnO}_{3}$ Heterostructures. Phys. Rev. Lett. 2010, 105, 027201.

(21) Borisevich, A. Y.; Chang, H. J.; Huijben, M.; Oxley, M. P.; Okamoto, S.; Niranjan, M. K.; Burton, J. D.; Tsymbal, E. Y.; Chu, Y. H.; Yu, P.; Ramesh, R.; Kalinin, S. V.; Pennycook, S. J. Suppression of Octahedral Tilts and Associated Changes in Electronic Properties at Epitaxial Oxide Heterostructure Interfaces. 
Phys. Rev. Lett. 2010, 105, 087204.

(22) Neumann, R. F.; Bahiana, M.; Binggeli, N. Magnetic Properties of $\mathrm{La}_{0.67} \mathrm{Sr}_{0.33} \mathrm{MnO}_{3} / \mathrm{BiFeO}_{3}(001)$ Heterojunctions: Chemically Abrupt vs. Atomic Intermixed Interface. EPL 2012, 100, 67002.

(23) Hatt, A. J.; Spaldin, N. A.; Ederer, C. Strain-Induced Isosymmetric Phase Transition in $\mathrm{BiFeO}_{3}$. Phys. Rev. B 2010, 81, 054109.

(24) Chu, Y. H.; Martin, L. W.; Holcomb, M. B.; Ramesh, R. Controlling Magnetism with Multiferroics. Mater. Today 2007, 10, 16-23.

(25) Zhang, J. X. He, Q.; Trassin, M.; Luo, W.; Yi, D.; Rossell, M. D.; Yu, P.; You, L.; Wang, C. H.; Kuo, C. Y.; Heron, J. T.; Hu, Z.; Zeches, R. J.; Lin, H. J.; Tanaka, A.; Chen, C. T.; Tjeng, L. H.; Chu, Y. H.; Ramesh, R. Microscopic Origin of the Giant Ferroelectric Polarization in Tetragonal-Like $\mathrm{BiFeO}_{3}$. Phys. Rev. Lett. 2011, 107, 147602.

(26) Sun, M. Y.; Li, P.; Jin, C.; Wang, L. Y.; Zheng, D. X.; Bai, H. L. Enhanced Exchange Bias in Fully Epitaxial $\mathrm{Fe}_{3} \mathrm{O}_{4} /$ Tetragonal-Like $\mathrm{BiFeO}_{3}$ Magnetoelectric Bilayers. EPL, 2014, 105, 17007.

(27) Kohn, W.; Sham, L. J. Self-Consistent Equations Including Exchange and Correlation Effects. Phys. Rev. 1965, 140, A1133-A1138.

(28) Blöchl, P. E. Projector Augmented-Wave Method. Phys. Rev. B 1994, 50, 17953-17979.

(29) Kresse, G.; Furthmüller, J. Efficient Iterative Schemes for Ab Initio Total-Energy Calculations Using a Plane-Wave Basis Set. Phys. Rev. B 1996, 54, 11169-11186. 
(30) Perdew, J. P.; Burke, K.; Ernzerhof, M. Generalized Gradient Approximation Made Simple. Phys. Rev. Lett. 1996, 77, 3865-3868.

(31) Wang, C.; Stojić, N.; Binggeli, N. Optimal Interface Doping at $\mathrm{La}_{2 / 3} \mathrm{Sr}_{1 / 3} \mathrm{MnO}_{3} / \mathrm{SrTiO}_{3}(001)$ Heterojunctions for Spintronic Applications. Appl. Phys. Lett. 2013, 102, 152414.

(32) Ferrari, V.; Pruneda, J. M.; Artacho, E. Density functional and half-metallicity in $\mathrm{La}_{2 / 3} \mathrm{Sr}_{1 / 3} \mathrm{MnO}_{3}$, phys. Stat. sol. (a) 2006, 203, 1437-1441.

(33) Tütüncü, H. M.; Srivastava, G. P. Electronic Structure and Lattice Dynamical Properties of Different Tetragonal Phases of $\mathrm{BiFeO}_{3}$. Phys. Rev. B 2008, 78, 235209.

(34) Singh, M. K.; Ryu, S.; Jang, H. M. Polarized Raman Scattering of Multiferroic $\mathrm{BiFeO}_{3}$ Thin Films with Pseudo-Tetragonal Symmetry. Phys. Rev. B 2005, 72, 132101.

(35) Sando, D.; Barthélémy, A.; Bibes, $\mathrm{M} . \mathrm{BiFeO}_{3}$ Epitaxial Thin Films and Devices: Past, Present and Future. J. Phys.: Condens. Matter 2014, 26, 473201.

(36) Wang, D. H.; Goh, W. C.; Ning, M.; Ong, C. K. Effect of Ba Doping on Magnetic, Ferroelectric, and Magnetoelectric Properties in Multiferroic $\mathrm{BiFeO}_{3}$ at Room Temperature. Appl. Phys. Lett. 2006, 88, 212907.

(37) Tsui, F.; Smoak, M. C.; Nath, T. K.; Eom, C. B. Strain-Dependent Magnetic Phase Diagram of Epitaxial $\mathrm{La}_{0.67} \mathrm{Sr}_{0.33} \mathrm{MnO}_{3}$ Thin Films. Appl. Phys. Lett. 2000, 76, 2421.

(38) Autieri, C.; Sanyal, B. Unusual Ferromagnetic $\mathrm{YMnO}_{3}$ Phase in 
$\mathrm{YMnO}_{3} / \mathrm{La}_{2 / 3} \mathrm{Sr}_{1 / 3} \mathrm{MnO}_{3}$ Heterostructures. New J. Phys. 2014, 16, 113031.

(39) Ranke, W.; Ritter, M.; Weiss, W. Crystal Structures and Growth Mechanism for Ultrathin Films of Ionic Compound Materials: $\mathrm{FeO}(111)$ on $\mathrm{Pt}(111)$. Phys. Rev. B 1999, 60, 1527-1530.

(40) Palai, R.; Katiyar, R. S.; Schmid, H.; Tissot, P.; Clark, S. J.; Robertson, J.; Redfern, S. A. T.; Catalan, G.; Scott, J. F.; $\beta$ Phase and $\gamma-\beta$ Metal-Insulator Transition in Multiferroic $\mathrm{BiFeO}_{3}$. Phys. Rev. B 2008, 77, 014110.

(41) Perdew, J. P.; Parr, R. G.; Levy, M.; Balduz Jr J. L. Density-Functional Theory for Fractional Particle Number: Derivative Discontinuities of the Energy. Phys. Rev. Lett. 1982, 49, 1691-1694.

(42) Maurice, J. L.; Pailloux, F.; Barthélémy, A.; Durand, O.; Imhoff, D.; Lyonnet, R.; Rocher, A.; Contour, J. P. Strain Relaxation in the Epitaxy of $\mathrm{La}_{2 / 3} \mathrm{Sr}_{1 / 3} \mathrm{MnO}_{3}$ Growth by Pulsed-Laser Deposition on $\mathrm{SrTiO}_{3}(001)$. Phil. Mag. 2003, 83, 3201-3224.

(43) Kuo, H. H.; Chen, L.; Ji, Y. Z.; Liu, H. J.; Chen, L. Q.; Chu, Y. H. Tuning Phase Stability of Complex Oxide Nanocrystals via Conjugation. Nano Lett. 2014, 14, 3314-3320. 


\section{Table of Contents Graphic (TOC)}

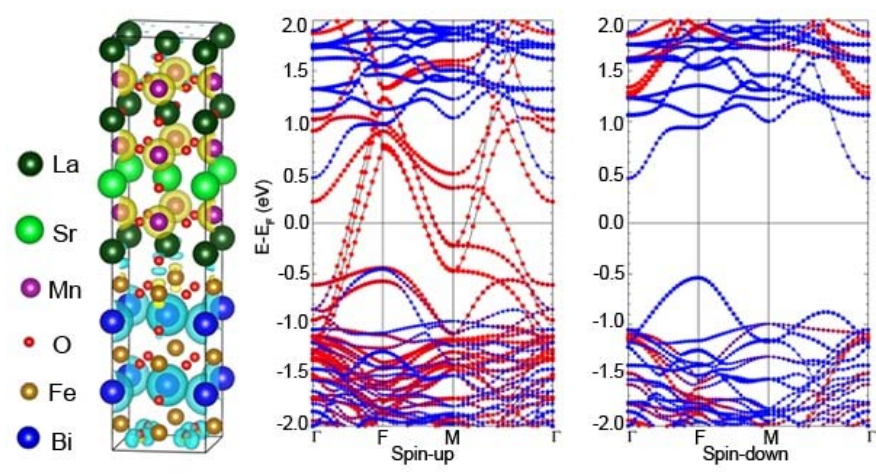

